\title{
Run II Physics at the Fermilab Tevatron and Advanced Analysis Methods
}

\author{
Pushpalatha C. Bhat ${ }^{\mathrm{a} *}$ \\ ${ }^{a}$ Fermi National Accelerator Laboratory ${ }^{\dagger}$ \\ P.O. Box 500, Batavia, IL 60510, USA
}

The Fermilab Tevatron has the unique opportunity to explore physics at the electroweak scale with the highest ever proton-antiproton collision energy of $\sqrt{s}=1.96 \mathrm{TeV}$ and unprecedented luminosity. About 20 times more data is expected to be collected during the first phase of the collider Run II which is in its second year of data-taking. The second phase of Run II, expected to begin in 2005, will increase the integrated luminosity to about 10-15 $\mathrm{fb}^{-1}$. Discovering a low mass Higgs boson and evidence for Supersymmetry or for other new physics beyond the Standard Model are the main physics goals for Run II. It is widely recognized that the use of advanced analysis methods will be crucial to achieve these goals. I discuss the current status of Run II at the Tevatron, prospects and foreseen applications of advanced analysis methods.

\section{INTRODUCTION}

The first phase of the second major protonantiproton collider run (Run IIa) is well underway at Fermilab. Major upgrades to the accelerator complex include a brand new $150 \mathrm{GeV}$ proton synchrotron called the Main Injector and a permanent magnet based antiproton recycler storage ring. The Main Injector enables 10 times more protons to be injected into the Tevatron, as compared to run I. The recycler helps recover the unused antiprotons from colliding beams of the Tevatron, store and reuse them in subsequent collisions. The collision energy is upgraded to $\sqrt{s}=1.96 \mathrm{TeV}$, up from $\sqrt{s}=1.80 \mathrm{TeV}$ in Run I.

The CDF and DØ experiments also underwent major upgrades in preparation for Run II[1]. The CDF detector had the inner tracker replaced, a plug calorimeter added and muon detectors upgraded. The DØ detector acquired a new central tracker with silicon microstrip and scintillating fiber tracking layers inside a $2 \mathrm{~T}$ solenoidal magnetic field and new scintillating fiber preshower detectors. The muon system has been upgraded with a new layer of scintillators in the central region and an all new forward muon system. A

\footnotetext{
*pushpa@fnal.gov †ंFermilab is operated by the Universities Research Association for the U.S. Department of Energy.
}

forward proton spectrometer has been added to enhance capabilities for diffractive physics. Both experiments required new trigger and data acquisition systems.

A rich harvest of physics is expected from an order of magnitude more data that is expected to be collected in Run IIa alone. The broad physics program consists of the study of Quantum Chromodynamics via the study of jets, (particularly a high statistics study with the high transverse energy jets), electroweak physics with the $\mathrm{W}$ and $\mathrm{Z}$ bosons, beauty and charm quark physics, top quark physics including the possible evidence and study of the electroweak production of single top, and searches for the Higgs boson and for signals of new physics beyond the Standard Model, notably, the signatures for supersymmetry, leptoquarks, technicolor or extra spatial dimensions.

In the following section, I give a short status report on the standard physics signals that are now being studied at the CDF and D $\varnothing$ experiments in an effort to understand the detectors and pursue studies on a wide range of physics topics. Then, I discuss, in the subsequent sections, the advanced analysis methods that are of import in improving the various aspects of physics analysis and prospects for exciting physics applications. 


\section{EARLY PHYSICS RESULTS FROM RUN II}

By the end of spring of 2002, after a year of running, the Tevatron had delivered about $55 \mathrm{pb}^{-1}$ each to the CDF and DØ experiments. Most of these data have been utilized to commission and tune the detectors. Some data have been used to look at some standard physics signals. The best way to evaluate the performance of the detectors and tune the event reconstruction algorithms and assess calibration errors in these early days of the run is to look at known signals in accessible mass regions. I present here some preliminary results [2] from such studies using Run II data.

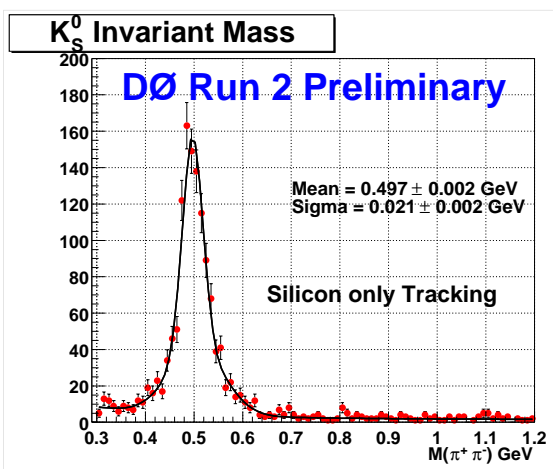

Figure 1. The $K_{s}^{0} \rightarrow \pi^{+} \pi^{-}$invariant mass spectrum using tracks from the Silicon detector.

Fig. 1 shows the invariant mass spectrum from two unlike sign tracks measured in the DØ Silicon detector only, which reveals the $K_{s}^{0} \rightarrow \pi^{+} \pi^{-}$ decays. Including measurements from the central fiber tracker improves the mass resolution to 5 $\mathrm{MeV}$. Better alignment of the sub-detectors using data is expected to provide further improvement in track parameter measurements.

The dimuon invariant mass spectrum showing the $J / \Psi$ and the $\Upsilon$ peaks using muons tracked and measured in the $\mathrm{D} \varnothing$ muon system alone and the cleaner $J / \Psi$ peak resulting after matching the
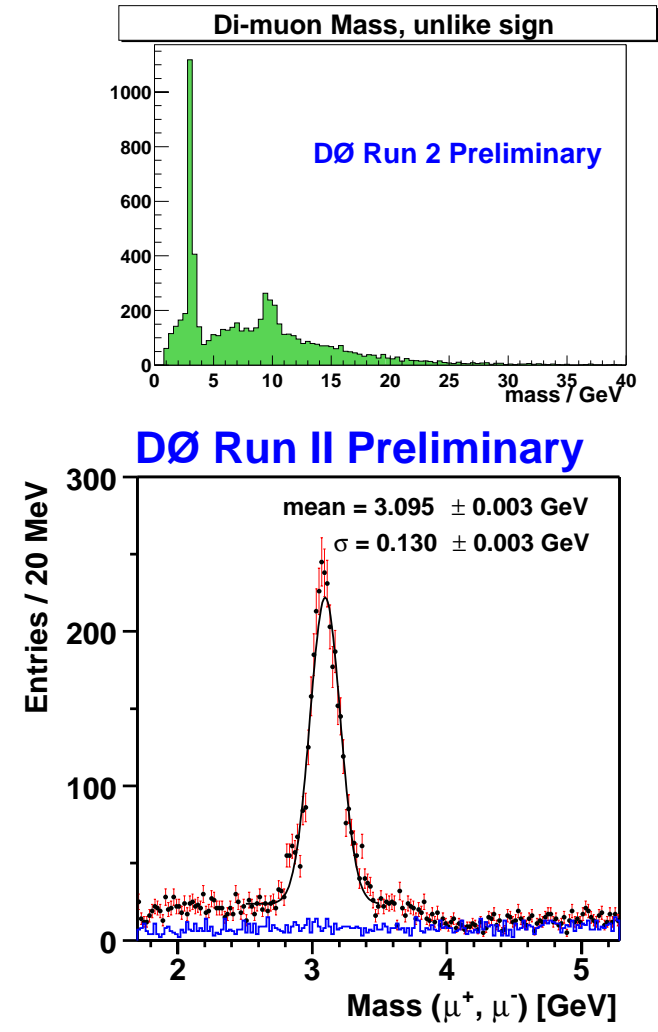

Figure 2. The di-muon invariant mass spectrum showing the $\mathrm{J} / \Psi$ and the $\Upsilon$ peaks (top). The di-muon invariant mass spectrum after matching muons found in the muon system with tracks from the central detector shows a clean signal of $\mathrm{J} / \Psi$ (bottom).

muons with the central detector tracks are displayed in Fig. 2.

The electroweak gauge bosons $(W, Z)$ are of paramount importance both in their own right and in inclusive signal and/or background channels in many interesting physics processes. The $Z \rightarrow l^{+} l^{-}$event samples serve as good calibration tools for lepton measurements. The invariant mass distributions of $Z \rightarrow e e$ from $\mathrm{D} \varnothing$ and $Z \rightarrow \mu \mu$ from CDF are shown in Figs. 3 and 4, respectively.

The inclusive $p_{T}$ spectrum of jets and the dijet mass spectrum measured in the $\mathrm{D} \varnothing$ calorimeter 


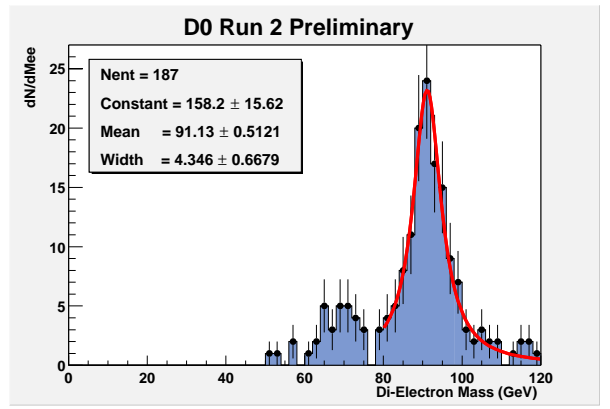

Figure 3. The di-electron invariant mass spectrum (from $\mathrm{D} \varnothing$ ) showing the $\mathrm{Z}$ boson peak.

in the central rapidity region of $|\eta|<0.5$ are shown in Fig. 5. The jet energy corrections used are preliminary. With the full Run II data-set the transverse energy distribution of the jets should extend to beyond $600 \mathrm{GeV}$.

Enormous progress is being made at both experiments in understanding calibration and corrections, and in tuning event reconstruction and particle identification algorithms. A large number of physics analyses are in progress.

\section{ADVANCED ANALYSIS METHODS}

Uncovering the signals of new physics in a hadron collider environment is extremely challenging because of a wide variety of processes that can mimic a given signature. Therefore, the use of advanced data analysis techniques are absolutely necessary for optimal separation of signal and background. The main data analysis tasks performed in HEP are particle identification, signal/background event classification, parameter estimation (precision measurements), functional approximation (fitting) and data-driven feature extraction or exploration. The best use of data is ensured only with multivariate treatment.

Suitable choice and representation of multivariate data are important first steps for a successful application. These could be labelled simply as intelligent pre-processing of data. In some applica-

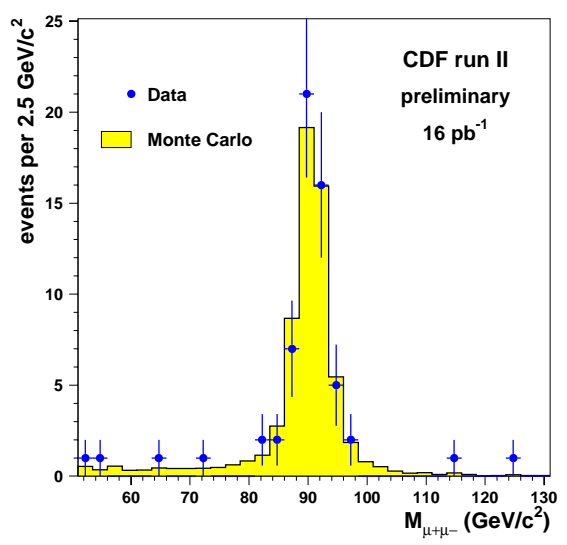

Figure 4. The $\mathrm{Z} \rightarrow \mu \mu$ signal from CDF Run II data [3].

tions this pre-processing might be the only necessary multivariate treatment of the data. The selection of variables for a given analysis application can be performed using the characteristic physics information or with algorithmic approach, employing, e.g., grid searches. Having selected a set of variables, one might like to apply a suitable transformation to the variables that would yield a representation of the data that most clearly exhibits certain desirable or "interesting" properties. That is, if $\mathbf{x}$ is the original multidimensional datum, then we seek $\mathbf{s}=\mathbf{f}(\mathbf{x})$ which has the desirable properties. If a linear transformation is employed, then, $\mathbf{s}=\mathbf{W} \mathbf{x}$, where $\mathbf{W}$ is the tranfer matrix.

The transformation of variables is equivalent to extracting a map $f: \mathrm{R}^{d} \rightarrow \mathrm{R}^{N}$. If $N<d$, then we would have effected a dimensionality reduction. There are nonlinear algorithms that use probability density estimation - histogramming, kernel-based methods and the methods of adaptive mixtures, and those that use stochastic optimization such as neural networks. For a review of these methods, see ref. [4]. In the following, I have chosen to discuss a few potentially interesting methods for HEP applications. 

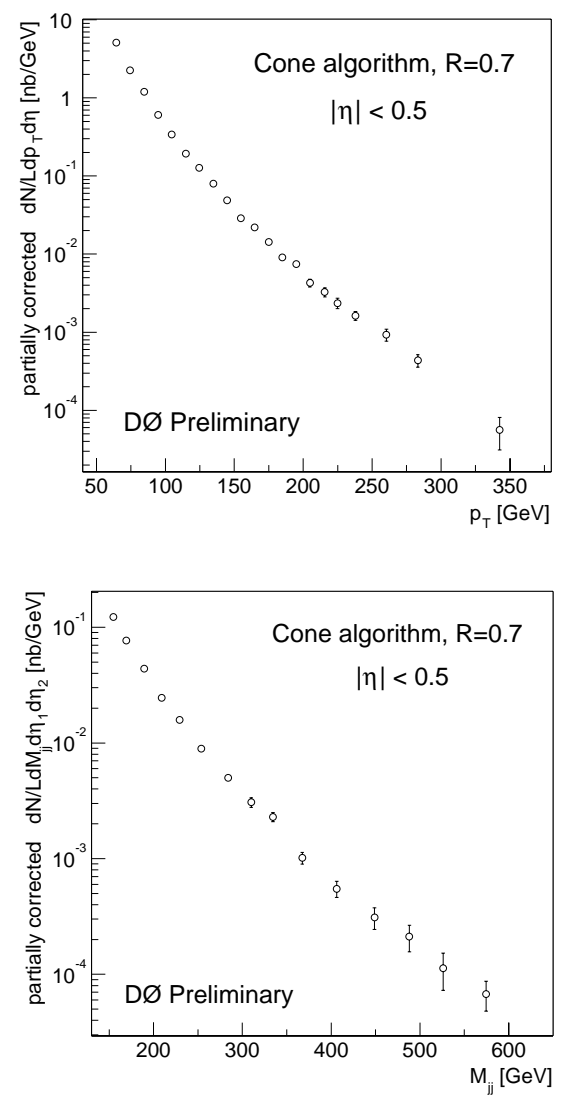

Figure 5. Inclusive jet $p_{T}$ spectrum (top) and dijet mass spectrum (bottom) from $\mathrm{D} \varnothing\left(\int L d t=\right.$ $\left.1.9 p b^{-1}\right)$.

\subsection{Grid Search, Principal and Indepen- dent Component Analysis}

Grid searches provide a systematic way of finding good variables and optimal cuts in multidimensional space, albeit not taking into account the correlations between variables. We developed a simple random grid search method [5] that can be used to compare the efficacy of variables as well as for a rapid search for optimal cuts for univariate classification. The results of such a grid search can be used as a benchmark to compare more sophisticated multivariate analyses.
To do a more advanced analysis, one would like suitably transformed variables as discussed earlier. Geometrically speaking, then, in a grid search one finds optimal cuts along the given coordinates while the Principal and Independent Component Analyses (PCA \& ICA) one finds interesting new directions (coordinates) in the multivariate space.

In the PCA algorithm (also known as Karhunen-Loeve transform or Hotelling transfor$\mathrm{m})$, the variance along the axes is used as the interesting feature while finding transformed variables. The new set of orthogonal basis is obtained by finding eigenvectors $\mathbf{u}_{\mathbf{i}}$ and eigenvalues $\lambda_{\mathbf{i}}$ as solutions of the equation,

$$
\mathbf{C u}_{\mathbf{i}}=\lambda_{\mathbf{i}} \mathbf{u}_{\mathbf{i}}
$$

where $\mathbf{C}=\mathbf{E}(\mathbf{x}-\overline{\mathbf{x}})(\mathbf{x}-\overline{\mathbf{x}})^{\mathbf{T}}$ is the covariance matrix of the data set $\mathbf{x}$. The transformation matrix, $\mathbf{W}$ has as its columns the eigenvectors and the transformed variables are $\mathbf{s}=\mathbf{W} \mathbf{x}$.

The ICA [6] is a relatively new technique, invented only in the past decade. It can be seen as a powerful extension of statistical factor analysis and PCA. The observed variables are assumed to be linear or nonlinear mixtures of unknown latent variables. The ICA technique enables transformation of data variables to extract these underlying statistically independent factors.

The ICA technique has been used in analyzing medical images, signal processing, and in the field of economics. Application to HEP would be a completely new exercise, which we have recently undertaken.

\subsection{Neural Networks and their Ensembles}

Artificial Neural Networks, though inspired from biology conceptually, are rigorous mathematical models for developing the map - $f: \mathrm{R}^{d} \rightarrow$ $\mathrm{R}^{N}$ where $N<<d$ and generally $N \sim 1$, without requiring a mathematical description of how the output(s) depend on the inputs.

Good generalization, that is good predictions for new inputs, is extremely important to minimize classification errors or to avoid over-fitting of data. The conventional methods for achieving good generalization have been (a) optimizing the size of the network given the training sample and (b) regularizing the training by penal- 
izing model complexity. But there are new and sophisticated approaches to achieve better generalization. (See e.g., [7].) And these involve using of ensembles of networks such as "committees" or "stacks." The basic concept in the usage of ensembles is to use many "nearly the best" networks with varying models (i.e., in architecture and input variables) rather than the "best" network, in ways that would help reduce the generalization error. It would be useful to arrive at rigorous or heuristic approaches to efficiently arrive at such ensembles.

\subsection{Self Organizing Maps (SOM)}

The self organizing map algorithm is an unsupervised technique which can be used for modelindependent exploration of data by finding cluster patterns in data. The idea of SOM was first introduced and developed by Kohonen in early 1980's (hence called Kohonen map, as well). The algorithm maps multidimensional feature space onto, usually, a 2 dimensional space with a lattice of nodes. Each node is associated with a vector of weight $\mathbf{w}$ of dimensionality $\mathbf{d}$ of the original space. An input vector is compared to all the weight vectors and assigned to the node that best matches the input.

\subsection{Support Vector Machines (SVM)}

The Support Vector Machines algorithm is a fairly new one. The main idea is to map the feature space into a space of sufficiently high dimensions $\left(f: \mathrm{R}^{d} \rightarrow \mathrm{R}^{N} ; N>>d\right)$ so that the optimal discriminating boundary between classes is a hyperplane and hence can be found using linear methods. Given the feature vector $\mathbf{x}$, the optimal hyperplane is $\mathbf{w} \cdot \mathbf{b}+\mathbf{x}$ where $\mathbf{w}$ is the unit vector normal to the hyperplane and $|b|$ is the distance of the plane from the origin.

For more details on the SVM method see contribution from Vaiciulis [8].

\subsection{Multivariate Analysis Issues}

The important issues to pay attention to in performing a multivariate analysis are the following:

- Choosing a set of variables without losing information
- Choosing the right method for the problem, which in many cases, has to be done by trying out a few methods.

- Controlling model complexity, i.e., keeping the number of free parameters in the multivariate model small compared to the sample size.

- Testing convergence of training in stochastic optimization algorithms, i.e., to have a good criteria to know when the training is optimal and cannot be improved further.

- Validating the learning or modeling, i.e., quantifying the correctness of modeling or goodness of learning, especially given a limited sample.

- Computational efficiency of the method and/or algorithm - it is important that the algorithm is computationally efficient so that the analysis can be repeated for many scenarios to ensure the robustness of the results.

\section{APPLICATIONS AND PROSPECTS}

The key factors responsible for the sweeping success of neural network (NN) algorithms for multivariate analysis are their power, ease of use and many successful applications in HEP. To cite a few examples from Run I Tevatron physics - (1) the top quark discovery at $\mathrm{D} \varnothing$ benefitted from comparisons of conventional analysis with results from NN analysis [9], (2) precision measurements of the top quark mass at $D \varnothing$ in lepton+jets and dilepton channels where the advanced methods helped reduce the statistical uncertainities by a factor of two, (3) top quark study in all-jets decay mode and searches for single top production at D $\varnothing$ and CDF, (4) world's best limit on first generation scalar leptoquark mass obtained by DØ. There are many spectacular applications of multivariate methods at LEP and HERA experiments. Some example applications and prospects have been presented in other talks at this workshop [10].

In 1990, I believed that multivariate methods would provide huge gains in top quark searches. 


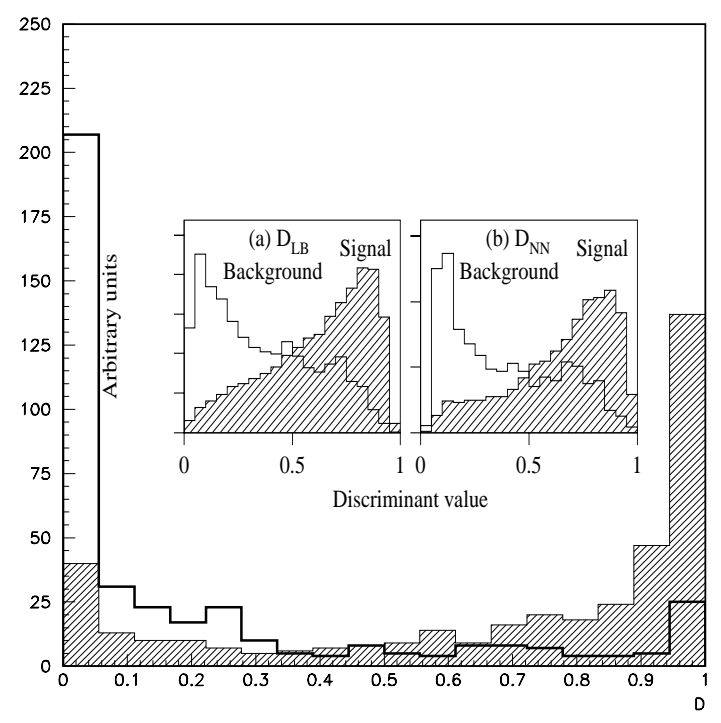

Figure 6. Likelihood discriminant distributions for signal (hatched histograms) and background events in $t \bar{t} \rightarrow$ lepton + jets channel using the new matrix element method [12]. The inset shows results from earlier analyses using a multivariate likelihood method (left) and NN (right).

We employed multivariate methods, at DØ, particularly neural networks, to optimize signal selection cuts that helped in top quark physics studies from discovery to precision measurements[11]. Using advanced multivariate and Bayesian methods, the DØ collaboration measured the top quark mass to be $173.6 \pm 5.6 \pm 6.0 \mathrm{GeV}$ in the lepton+jets channel with a better than expected statistical precision of $5.6 \mathrm{GeV}$. This extraordinary feat in Run I of precision top quark mass measurement has now been surpassed by exploiting probabilistic information for each event in the matrix element method [12] using the same Run I sample. The comparisons of discrimination between signal and background are shown in Fig. 6. The new measurement yields a top mass of $179.9 \mathrm{GeV}$ with a statistical uncertainty of $3.6 \mathrm{GeV}$. Run II, with an expected yield of the order of $500 \mathrm{~b}-$ tagged $t \bar{t}$ events in lepton+jets final state alone per $f b^{-1}$ recorded, ushers in an era of a variety of precision measurements in top quark physics.

Topping the list of interesting searches in Run II is that for the Higgs boson. The Higgs mechanism is one vital piece of the standard model that still awaits experimental evidence. Therefore, the Higgs boson would be the most sought after particle in Run II. The discovery of a SMlike Higgs boson will lend credence to the popular theories of the origin of mass. The Tevatron Run II Higgs working group explored the discovery reach for the Higgs boson and the results are shown in Fig. 7. The details of the analysis are described in published papers and the working group report [13]. There are valid and intriguing reasons for the prevailing optimism that the Higgs boson and Supersymmetry may be around the corner. The most favored Higgs boson mass from constraints from precision measurements is in the neighborhood of $100 \mathrm{GeV}$. In most SUSY models, the Higgs mass is below $150 \mathrm{GeV}$. The Tevatron, although, has good prospects for discovering a low mass Higgs boson, it is not going to be easy. It is important to emphasize, however, that the discovery reach at a given mass requires half the integrated luminosity if multivariate methods are adopted instead of conventional univariate methods.

Searches for signatures from new physics beyond the Standard Model such as leptoquark production, supersymmetry or technicolor, are also employing multivariate methods in various stages of Run II data analysis. Advanced multivariate and statistical techniques [14] form a powerful combination that enable optimal use of data, consistent treatment of uncertainties and meaningful model comparisons.

\section{SUMMARY}

Run II is well underway at Fermilab. Early physics results from the upgraded CDF and D experiments promise an exciting physics program in the years ahead. A new era of precision measurements in standard model physics and of exciting opportunities to discover the agent(s) of electroweak symmetry breaking and new physics beyond the standard model has commenced. There 


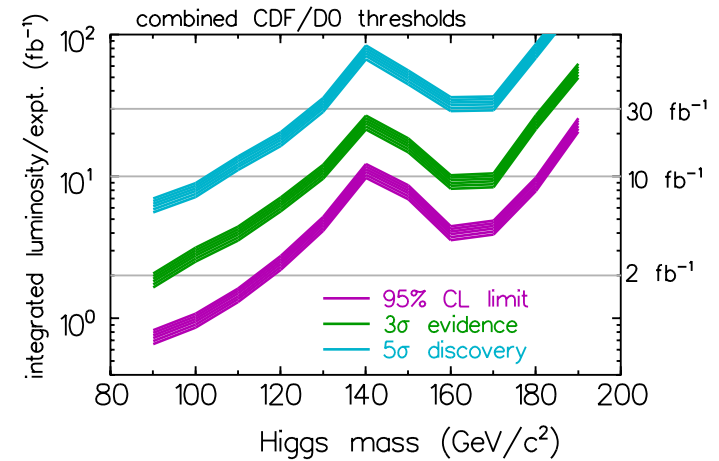

Figure 7. The required integrated luminosity for $5 \sigma, 3 \sigma$ observation or $95 \%$ C.L. exclusion of the SM Higgs boson in Run II. For details of the analysis see ref. [13].

are strong theoretical motivations for new discoveries and valid reasons for the prevailing optimis$\mathrm{m}$. The multivariate methods will provide sensitivity to new particles with masses beyond the reach of conventional methods of analysis based on univariate cuts. Advanced statistical methods adopting a fully probabilistic approach will enable better precision measurements and better explorations of model parameters. In short, the use of advanced multivariate and statistical techniques will enable new discoveries and produce results with better precision, robustness and clarity.

\section{REFERENCES}

1. CDF Collaboration, "The CDF II Detector Technical Design Report," FERMILABPUB-96/390-E (1996); DØ Collaboration, " The D0 upgrade: The Detector and its physics," FERMILAB-PUB-96-357-E (1996).

2. M. Verzocchi (for DØ Collaboration), Proceedings of the 37th Rencontres de Moriond on Electroweak Interactions and Unified Theories, Les Arcs, France, March 2002, hep-ex/0205049; M. Rescigno, ibid, hepex/0205092.

3. F. Bedeschi, Proceedings of the 31st International Conference on High En- ergy Physics, Amsterdam, July 2002, http://www.ichep02.nl.

4. P.C. Bhat, Proceedings of the VII International Workshop on Advanced Computing \& Analysis Techniques in Physics Research (ACAT2000), Batavia, IL (AIP 2001) p. 22

5. H. Prosper et al., Proceedings of the International Conference on Computing in High Energy Physics, 1995, Rio de Janeiro, Brazil (World Scientific, 1996).

6. A. Hyvärinen, K. Karhunen and E. Oja, "Independent Component Analysis," John Wiley and Sons, 2001, Also see http://www.cis.hut.fi/projects/ics/.

7. C. Bishop, "Neural Networks and Pattern Recognition," Oxford University Press (1995). There are many other books, papers and web resources on neural networks.

8. A. Vaiciulis, these proceedings.

9. P.C. Bhat, Proceedings of the 8th meeting of the Division of Particles \& Fields of the American Physical Society, Albuquerque, NM, USA (World Scientific, 1994) p.705; P.C. Bhat, Proceedings of the 10th Topical Workshop on Proton-Antiproton Collider Physics, Batavia, IL, USA (AIP, 1995) p.308.

10. E. Boos, L. Dudko and D. Smirnov, these proceedings; L. Litov, ibid; J.-C. Prévotet, et al., ibid; A. Badalà, et al., ibid; J. Zimmermann, C. Kiesling and P. Holl, ibid.

11. P. C. Bhat, H.B. Prosper and S.S. Snyder, Int. J. Mod. Phys. 135113 (1998) and references therein.

12. J. Estrada, Proceedings of the DPF Meeting of the American Physics Society, May 24-28, 2002, Williamsburg, Virginia.

13. P.C. Bhat, R. Gilmartin and H.B. Prosper, Phys. Rev. D. 62074022 (2000); T. Han, et al., Phys.Rev. D. 59, 093001 (1999); Tevatron Run II Higgs Group Report hep-ph0010338.

14. Fermilab Advanced Analysis Methods Group, http://projects.fnal.gov/run2aag/. 